\title{
„Wir möchten mit den Herstellern ins Gespräch kommen“
}

Deutschland befindet sich bereits mitten in der Energiewende, die Senkung des Energieverbrauchs durch mehr Energieeffizienz steht dabei schon länger auf der politischen Agenda, schließlich ist die sauberste und billigste Energie die, die gar nicht erst verbraucht wird. Damit die Energiewende ein Erfolg wird, müssen Strom und Wärme noch effizienter genutzt werden. Wie kann dies im Gesundheitswesen und besonders in der Radiologie gelingen? Und welche Anforderungen sollten radiologische Großgeräte und ihre Hersteller in der Industrie erfüllen, um nachhaltig zu sein? Darüber haben wir in Teil 2 unserer Interviewreihe \#NachhaltigeRadiologie zum Thema Nachhaltigkeit in der Radiologie mit Professor Andreas G. Schreyer und Dr. Kerstin Jungnickel gesprochen. Professor Schreyer ist Institutsdirektor und Chefarzt am Institut für Diagnostische und Interventionelle Radiologie am Universitätsklinikum Brandenburg an der Havel. Dr. Kerstin Jungnickel ist als Medizinphysikerin am Klinikum Magdeburg tätig.

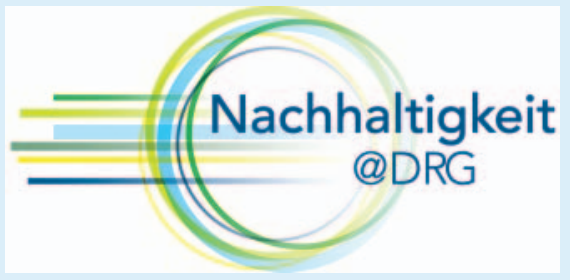

Beide sind Mitglieder der Kommission Nachhaltigkeit@DRG.

Bei der privaten Anschaffung von Haushaltsgeräten sind die Energieeffizienzklassen bereits ein wichtiges Auswahlkriterium. Energieeffizienz bei radiologischen (Groß-)Geräten scheint hingegen kein allzu großes Thema zu sein. Oder täuscht der Eindruck?

Dr. Jungnickel: In der Radiologie wird Wert auf eine gute Ausstattung der Geräte gelegt. Insbesondere in den Unikliniken wird bei Ausschreibungen auf optimale Leistung geachtet (schnelle Bildgebung, hohe Bildqualität), was üblicherweise mit einem höheren Energieverbrauch einhergeht. In der Niederlassung werden gute, solide Geräte gewählt, die zudem günstig sind. Auch da spielt die Energieeffizienz (fast) keine Rolle. Einzig in der Ausschreibung findet man die Abfrage nach Energieverbrauch (meist ohne Wertung und ohne feste Kriterien) und die Frage nach den Kosten des Wartungsvertrags, also was das Gerät in den nächsten Jahren an Folgekosten erzeugt. Allerdings bezieht sich das üblicherweise nicht auf den Energieverbrauch. Die Bewertung der Nachhaltigkeit von Geräten (Energieeffizienz, Reparatur- und Recyclingfähigkeit) könnte durch standardisierte Ausschreibungstexte mit verpflichtenden Umweltsiegeln deutlich vereinfacht werden. Energielabel gibt es leider nicht bei medizinischen Großgeräten. Das wäre eine große Hilfe. 
Professor Schreyer: Genau an dem Punkt Effizienz will die Kommission Nachhaltigkeit@DRG künftig helfend und beratend ansetzen. Der Gerätekauf in der Radiologie kann nicht mit dem Kauf eines Kühlschranks verglichen werden. Bei meiner letzten CT-Neubeschaffung habe ich zum Beispiel realisiert, dass teilweise bis zu 20 Prozent der Gerätekosten noch einmal zusätzlich durch neue Kühl- und Klimatechnik anfallen. Neben den hohen primären Investitionen führt das auch zu einem zusätzlich hohen Energieverbrauch. Das Thema wird dann noch viel komplexer, wenn man bedenkt, dass die Herstellung von Großgeräten, gerade bei MRT, ebenfalls sehr viele Ressourcen verbraucht. Die CO2-Bilanz wird nicht nur durch den täglichen Energiebedarf beeinflusst - gerade die Geräteherstellung ist ein gewaltiger Malus beim Thema CO2. Da gäbe es dann eine Parallele zu Haushaltsgeräten, wenn beispielsweise alle par Jahre ein neuer Kühlschrank gekauft wird aufgrund der besseren Energieeffizienz und dabei die mit der Herstellung einhergehende CO2-Belastung komplett ignoriert wird. Hier sollte man beim Kauf zum Beispiel bedenken, ob ein refurbished-CT oder MRT nicht vielleicht ökologisch und ökonomisch besser wären oder das Upgrade eines vorhandenen MRT ökologische Vorteile hat. Ein CT oder MRT einer hohen Leistungsklasse braucht in der Regel mehr Energie, macht aber meist auch bessere Bilder. Die Herausforderung wird also sein, dass wir hier eine Zusammenarbeit von Radiologie, Physik und Industrie erreichen, um eine realistische Effizienzklasse innerhalb der Leistungsklassen zu erstellen, die unter anderem auch die Upgrade- beziehungsweise RecyclingFähigkeit der Geräte berücksichtigt. Nur den Energieverbrauch der Bildqualität gegenüberzustellen, wäre falsch und zu kurz gegriffen. Dieses gesamtheitliche Effizienzlabel sehe ich als unser großes mittelfristiges Ziel, an dem wir als Kommission Nachhaltigkeit@DRG arbeiten sollten. Denn nur mit dieser Objektivierung können wir die Gerätehersteller in die korrekte Richtung leiten, wenn wir diese Label dann künftig in Ausschreibungen nutzen.
In unserer Interviewreihe \#NachhaltigeRadiologie fragen wir Akteurinnen und Akteure aus dem Gesundheitswesen nach ihren Ideen und Strategien für mehr Nachhaltigkeit in ihrem Tätigkeitsbereich. Wir wollen von ihnen auch wissen, wie vor allem die Radiologie nachhaltiger werden kann. Weitere Interviews dieser Reihe finden Sie unter www.nachhaltigkeit.drg.de.

Wie hoch ist der Energieverbrauch von radiologischen Großgeräten, etwa von Computer-/Magnetresonanztomografen, eigentlich?

Dr. Jungnickel: Der Energieverbrauch von radiologischen Großgeräten wurde in einem sehr schönen Paper von Heye et al. untersucht und 2020 in Radiology publiziert. Demnach verbraucht ein CT jährlich ca. 26000 kWh, ein MRT 134000 kWh. Zum Vergleich, ein 4-Personen-Haushalt in Deutschland benötigt im Schnitt ca. 3000 kWh im Jahr. Die Analyse von Heye zeigt zudem, dass 2/3 der Energie beim CT im unproduktiven Warte-Zustand verbraucht werden und beim MRT 1/3 der Energie im ausgeschalteten Zustand allein für die konstante Helium-Kühlung aufgewendet wird. Da gibt es auch herstellerseitig noch Luft nach oben.

Professor Schreyer: Umgerechnet sind das ökonomisch nur für die Energiekosten über $4000 €$ p.a. für einen CT-Scanner und $20660 €$ p. a. für ein MRT-Gerät. Wenn man die Gesamtenergiekosten für die Herstellung und Benutzung für eine einzelne MR-Studie herunterrechnet, entspricht eine einzige MRT-Untersuchung dem Verbrauch einer Klimaanlage über einen Tag in einem großen Haus oder der Entsalzung von 26500 Litern Meerwasser.

Erkennen Sie bei diesen Geräten in den letzten Jahren eine technische Weiterentwicklung hin zu mehr Energieeffizienz und Nachhaltigkeit?

Dr. Jungnickel: Beim MRT werben die Hersteller schon seit einigen Jahren mit immer geringerem Helium-Verbrauch. Das sehen wir bei Nachhaltigkeit und Energieeffizienz weniger. Genau dazu möchten wir mit den Herstellern ins Gespräch kommen.

Professor Schreyer: Erfreulicherweise gibt es bei den Herstellern schon Verantwortliche in der Entwicklung, die für Nachhaltigkeit zuständig sind. Doch durch die fehlende Vergleichbarkeit von verschiedenen technischen Ansätzen und Energieeffizienz-Kriterien (Stichwort „Gesamtheitliche Effizienzlevel“ aus Frage 1) ist man gegenwärtig versucht, zum Teil auch so etwas wie „Greenwashing“ zu vermuten. Aus Marketing-Gründen können die Geräte schnell und einfach grün oder blau angemalt werden, auch Heliumeffizienz ist häufig relativ und dem ökonomischen Druck des immer teurer werdenden Edelgases geschuldet. Als Optimist glaube ich zwar, dass sich langsam bezüglich Energieeffizienz etwas tut das Thema ist aber noch nicht in den absoluten Fokus gerückt. Daher ist es unser Job als verantwortungsbewusste Fachgesellschaft, den Geräteherstellern unsere Wünsche und Prioritäten bezüglich Nachhaltigkeit mit sanftem und klar formuliertem Druck mitzuteilen.

Um mehr Energieeffizienz und Nachhaltigkeit in radiologischen Praxen und Klinikabteilungen zu erreichen, muss man die Geräte als solche, aber sicherlich auch deren Handhabung sowie die übergeordneten Arbeits-/Versorgungsprozesse in den Blick nehmen. Welche Potenziale sehen Sie hier?

Dr. Jungnickel: Auch die Beschäftigten können den Energieverbrauch senken. Monitore und Rechner können nachts ebenso ausgeschaltet werden wie die Großgeräte, die nicht für den Notfall gebraucht werden. Auch die Klimaanlagen können nachts ihre Leistung reduzieren und müssen die Räume nicht so stark herunterkühlen, da weder Personen noch Maschinen Wärme abgeben.

Professor Schreyer: Zusätzlich kann ich mir vorstellen, dass durch intelligente Automatisierung der Energieverbrauch zusätzlich reduziert werden kann. Eine intelligente Raum- und Gerätekühlung könnte sich zum Beispiel an der tatsächlich anfallenden Untersuchungszahl orientieren. Ein weiterer wichtiger Punkt ist für mich die Datenspeicherung. Man muss sich einmal vor Augen halten, dass weltweit die Data-Centers und 
Server-Farmen zur Datenspeicherung die gleiche Menge CO2 erzeugen wie der weltweite Flugverkehr. CT-Datensätze für eine Untersuchung haben von durchschnittlich 66 Mbyte (2011) auf nun 160 Mbyte zugenommen. Die Radiologie ist weltweit in der Medizin der größte Datenerzeuger. Hier liegt für mich weiteres Potenzial, die Datenmengen in der Speicherung nicht ausufern zu lassen. Zusätzlich würden sogenannte „abbreviated protocols", also MRT- und CTProtokolle, die sich auf das medizinisch Notwendige beschränken, zu einer nachhaltigen CO2-Reduktion führen.

Mehr Nachhaltigkeit durch Energieeffizienz und Klimaschutz - das ist ein wichtiges Ziel, das aber auch finanziert werden muss. Wie schätzen Sie die Kosten im Zusammenhang mit der Modernisierung radiologischer Großgeräte ein?

Dr. Jungnickel: Nachhaltige Technik muss nicht teurer sein. Außerdem spart man mit einem geringeren Energieverbrauch automatisch Geld. Perfekt wäre natürlich die Berücksichtigung der Nachhaltigkeit schon zu Baubeginn einer Klinik oder Praxis, da dann auch Synergieeffekte genutzt würden, zum Beispiel könnten mit der Abwärme der Klimaanlage andere Bereiche geheizt werden.

Professor Schreyer: Wie eingangs erwähnt, erweisen wir der Nachhaltigkeit einen Bärendienst, wenn wir jetzt kurzfristig alle Großgeräte gegen die neuen etwas energieeffizienteren Versionen austauschen, da auch die Geräteherstellung in hohem
Maße Ressourcen verbraucht. Doch bei Neuinvestitionen muss es unsere Forderung und unser Anspruch an die Industrie sein, dass alle Geräte in Herstellung und täglichem Verbrauch hocheffizient werden. Besonders der immer noch immense Energieverbrauch der nicht genutzten Geräte nachts und an Wochenenden, der laut der Analyse von Heye bis zu 2/3 des Gesamtverbrauches ausmacht, ist erschreckend. Hier sollten kurzfristige Nachbesserungen eingefordert werden. Nachhaltige Technik muss und vor allem darf nicht teuer sein wir müssen diese Eigenschaften gemeinsam als selbstverständliches Feature, auf das wir beim Kauf achten, einfordern.

Wir danken Ihnen für das Gespräch, Herr Professor Schreyer und Frau Dr. Jungnickel! 